\title{
ZWISCHENRUF
}

\section{Rolle rückwärts in der Politikberatung?}

Wissenschaftliche Politikberatung ist im Laufe der letzten Jahrzehnte ein weites Feld geworden, in dem die Technikfolgenabschätzung eine unter vielen Formen ist. Sie bekommt in Deutschland nun weiteren Zuwachs: die Nationale Akademie, deren Gründung als relativ kompliziertes Konstrukt unter Führung der „Deutschen Akademie der Naturforscher Leopoldina“ im Februar bekannt gegeben wurde. Diese neue Akademie soll in Kooperation mit anderen Akademien unter dem Oberbegriff wissenschaftsbasierte Politikberatung gesellschaftliche Zukunftsthemen benennen, bearbeiten und der Öffentlichkeit sowie politischen Entscheidungsträgern in geeigneter Form vermitteln. Weiterhin soll sie eine wirkungsvolle Vertretung der in Deutschland tätigen Wissenschaftler in internationalen Gremien wahrnehmen.

Die Geburt dieser neuen Institution war schwierig. Die föderale Struktur der deutschen Forschungslandschaft bildete dabei ein fast unüberwindliches Hindernis, worüber auch öffentlich viel berichtet und gestritten wurde. Zwei weitere Aspekte wurden jedoch kaum thematisiert: Nach welchem Modell soll Politikberatung dort erfolgen? Und welches Verständnis zum Verhältnis zwischen Wissenschaft, Politik und Gesellschaft wird hierbei zugrunde gelegt?

Die Technikfolgenabschätzung hat mittlerweile etwa 40 Jahre Erfahrung im Feld der Politikberatung, sich dabei mehrfach gewandelt und entscheidend weiterentwickelt. Vor dem Hintergrund dieser Erfahrungen sei es gestattet, einige Anfragen in konstruktiver Absicht an die Nationale Akademie zu richten und diese zur Diskussion zu stellen. Diese Fragen lauten:

(1) Was bedeutet es, wenn die prominente Befassung mit gesellschaftlichen Zukunftsthemen federführend an die Leopoldina als eine Gesellschaft der Naturforscher übergeben wird? Naturforscher sind bekanntermaßen Experten für die Natur, die sie erforschen. Ob sie aber auch simultan Experten für die Zukunft der Gesellschaft sind, ist nicht so ohne Weiteres einsehbar. Zwar sollen Technik-, Geistes- und Sozialwissenschaftler einbezogen werden, die Federführung liegt aber bei Naturforschern. $\mathrm{Ob}$ es gelingt, die plurale Vielfalt der wissenschaftlichen Disziplinen und die je unterschiedlichen Perspektiven und Wissensbestände zusammenzuführen, wird von den Details der Arbeitsweise der Akademie abhängen.

(2) Aus den Verlautbarungen der Politik ist die Erwartung herauszuhören, dass im Rahmen der Nationalen Akademie die „eine“ Stimme der Wissenschaft zu gesellschaftlichen Zukunftsfragen formuliert wird. Diese wird es jedoch nicht geben, da kontroverse Werthaltungen, Menschenbilder, gesellschaftliche Gegenwartsdiagnosen und Zukunftsentwürfe ihren Platz keineswegs nur in Politik und Gesellschaft haben, sondern sich durch die Wissenschaft hindurchziehen. In vielen gesellschaftlich relevanten Feldern, man denke z. B. an die Energieversorgung der Zukunft, ist das Expertendilemma bekannt: Es gibt unterschiedliche und teils sich diametral widersprechende Expertenmeinungen, auch innerhalb einer Disziplin. Das, worauf Wissenschaftler sich einigen, wird in der Regel in Form von Lehrbuchwissen kodifiziert. Mit Lehrbuchwissen jedoch sind keine Positionen zur Energieversorgung der Zukunft, zur ethischen Rechtfertigbarkeit der Stammzellforschung oder zur „technischen Verbesserung“ des Menschen zu begründen. Selbst wenn die eine Stimme der Wissenschaft gefunden werden sollte, wird sie nicht viel Relevantes zu gesellschaftlichen Zukunftsthemen aussagen; wenn die Akademie sich in relevanter Weise zu gesellschaftlichen Zukunftsfragen äußern will und soll, wird sie mit entsprechenden Konflikten umgehen müssen. Man kann gespannt sein, wie die Nationale Akademie mit dieser Herausforderung umgehen wird.

(3) In vielen empirischen Untersuchungen zur Wirksamkeit wissenschaftlicher Politikberatung wird immer wieder betont, wie entscheidend die Kommunikation zwischen Wissenschaft und Politik „im Prozess“ ist. In der „klassischen“ Arbeitsweise von Akademien, die von wissenschaftlichen Expertengruppen getragen werden, ist diese Kommunikation „zwischendurch“ zwar nicht prinzipiell ausgeschlossen, kommt aber in der Praxis bislang kaum vor. Hier besteht für die Leopoldina die Herausforderung, neue Modelle akademischer Arbeit zu entwickeln und auf Erfahrungen aus der Politikberatung konstruktiv aufzubauen.

(4) Schließlich lässt die doppelte Zielsetzung von Politikberatung und Interessenvertretung in einer Institution aufhorchen. Wissenschaftliche Experten sind in der Regel auch Interessenvertreter in eigener Sache. Dies ist keineswegs verwerflich, sondern gehört sogar zu ihren Pflichten. Wenn Politikberatung in gesellschaftlichen Zukunftsfragen und Interessenvertretung der Wissenschaft in einer Institution zusammengeführt sind, könnten die Versuchungen groß sein, die Politikberatung nach den eigenen partikularen Interessen auszurichten - zumindest könnte nach außen rasch dieser Eindruck entstehen. Die Nationale Akademie wird sehr darauf achten müssen, in der Öffentlichkeit nicht den Anschein zu erwecken, dass sie Politikberatung zu gesellschaftlichen Zukunftsfragen als Interessenvertretung in eigener Sache betreibt.

Allen diesen Punkten kann durch ein entsprechendes „Design“ der Projekte und durch institutionelle Vorkehrungen begegnet werden. Eine „Rolle rückwärts“ hinein in technokratische Politikberatungsmodelle unter Ignorierung der Erfahrungen der letzten Jahrzehnte mit vielen etablierten und pragmatischen Formen „pragmatistischer Politikberatung“" (Habermas) wäre fatal und würde den Erfolg der Nationalen Akademie gefährden. 